with Certain Functional Impairments (LSS) and require PA with their basic daily needs for more than $20 \mathrm{~h}$ a week. The aim of the study was to investigate how the LSS Act provides possibilities to work for people entitled to measures for special support and special service.

Method Cross-sectional analysis based on data from the Swedish Social Insurance Agency and from a questionnaire survey of a sample of people entitled to PA (total of 15515). The response rate was $67 \%$.

Results Generally more men (27\%) than women (21\%) considered that PA is a prerequisite for their ability to work. Among those who were mentally retarded, were autistic or had a condition resembling autism (group 1), 33\% responded positively. Persons belonging to the group with considerable and permanent, intellectual functional impairment after brain damage in adulthood (group 2), experienced least possibilities to work (11\%). Among those with other lasting physical or mental functional impairments (group 3), 22\% experienced that PA gave them a possibility to work. Of those who had responded positively, 25\% were born in Sweden and 22\% were born abroad.

Conclusions Personal assistance seems to provide possibilities of active participation in the labour market for persons with substantial and permanent disability.

\section{CHARACTERISATION OF WET WORK AND GLOVE USE IN HEALTHCARE OCCUPATIONS}

Michael Humann, Abbas Virji, Xiaoming Liang, Marcia Stanton, Aleksandr Stefaniak, Paul Henneberger. National Institute for Occupational Safety and Health, Division of Respiratory Disease Studies, Morgantown, WV, USA

\subsection{6/oemed-2014-102362.37}

Objectives Wet work (contact and/or use of liquids) could lead to skin exposures to chemical irritants and sensitizers among healthcare workers. The objective of this study was to characterise the frequency and duration of glove use when wet work was performed by healthcare workers.

Method Direct observational studies were conducted from 2009 to 2011 at five hospitals on selected healthcare occupations. Information on tasks, chemical product use, and glove use was collected at five-minute intervals by trained research technicians using a standardised data collection form.

Results Between five and 51 person-days were observed for each occupation. Any glove use during wet work ranged from $62 \%$ to $100 \%$ of person-days for occupations with more than 10 person-days observed. Endoscopy technicians had the highest proportion of time of glove use when wet work was observed $(1845 / 2055 \min =90 \%)$, followed by medical equipment preparers, dental assistants, and housekeepers $(1645 / 1950 \mathrm{~min}=$ $84 \%, 315 / 395 \mathrm{~min}=80 \%$, and $6090 / 7720 \mathrm{~min}=79 \%$ respectively). Floor strippers/waxers $(585 / 1225 \mathrm{~min}=48 \%)$, respiratory therapists $(65 / 160 \mathrm{~min}=41 \%)$, and clinical laboratory technicians $(10 / 60 \mathrm{~min}=17 \%)$ had lower proportions of time of glove use. When a sensitizer was used during wet work, the proportion of time of glove use increased among all healthcare occupations with adequate data.

Conclusions This analysis demonstrates that the duration of wet work and glove use vary by healthcare occupation. This assessment will be valuable for developing health and safety training programs and identifying possible avenues for intervention.
Eve Bourgkard, Régis Colin, Michel Grzebyk, Isabelle Urmes, Guy Hedelin. INRS, Vandoeuvre-Lès-Nancy, France

\subsection{6/oemed-2014-102362.38}

Objectives To describe the mortality of sewage workers from Paris (France).

Method The cohort of 1594 Paris sewage workers since 1970 was set up in 2010 and followed-up on mortality from 1970 to 2010. Vital status and causes of death were determined by matching with national databases. Standardised Mortality Ratios (SMRs) were computed using local death rates by causes of death and 10-year employment duration classes. Data are currently analysed using relative survival techniques. This study was approved by the national ethic comity.

Results Statistically significant mortality excess was observed for all causes ( $\mathrm{SMR}=1.34,778$ cases) and for cancer $(\mathrm{SMR}=1.49$, 337 cases). SMRs were also statistically greater than 1 for malignant $(\mathrm{SMR}=1.74,22$ cases) and non-malignant ( $\mathrm{SMR}=1.77,43$ cases) liver diseases, lung cancer ( $\mathrm{SMR}=1.59,97$ cases), oesophagus cancer ( $\mathrm{SMR}=2.35,28$ cases), all alcohol-related diseases ( $\mathrm{SMR}=1.78,128$ cases), and suicide ( $\mathrm{SMR}=3.64,22$ cases). Greater than 1 but not statistically significant SMRs were observed for infectious diseases and respiratory infectious diseases. The mortality from several diseases (all causes, all cancer, oesophagus cancer, lung cancer, chronic liver diseases, all alcohol-related diseases, and infectious diseases) increased with employment duration as a sewer worker. Except for lung cancer, the SMR for smoking-related diseases was not statistically greater than 1. Results of survival analysis are in progress.

Conclusions The increase mortality observed for lung cancer and infectious diseases with employment duration suggests possible occupational health effect among sewer workers. Conclusions will be completed from the survival analysis.

\section{OCCUPATIONAL RISK FACTORS FOR ENDOMETRIOSIS AMONG FLIGHT ATTENDANTS}

Candice Johnson, Barbara Grajewski, Christina Lawson, Elizabeth Whelan, Stephen Bertke, Chih-Yu Tseng. National Institute for Occupational Safety and Health, Cincinnati, OH, USA

\subsection{6/oemed-2014-102362.39}

Objectives Previous studies suggest that flight attendants could have a higher risk for endometriosis than women in other occupations. Our objectives were to compare the rate of endometriosis among flight attendants to the rate in a comparison group of teachers, and to investigate occupational risk factors for endometriosis among flight attendants.

Method We included 1780 flight attendants and 240 teachers aged 18-45 at enrollment. Endometriosis diagnosis was selfreported via telephone interview, and records of individual flights were retrieved from airlines to obtain work schedules and assess exposures for flight attendants. Cox regression was used to estimate odds ratios (OR) and 95\% confidence intervals (CI) for associations between exposures and endometriosis, adjusting for body mass index at interview and using age as time scale.

Results Flight attendants were no more likely to report endometriosis than teachers (adjusted OR 1.3, 95\% CI 0.7-2.3). Among flight attendants, there were no clear trends between yearly cosmic radiation exposure, hours worked during normal sleeping hours, or ergonomic factors and endometriosis. A greater 